\title{
The low responder, hypogonadotropic hypogonadism female patient in IVF: do not give up!
}

\author{
Nathan Lewit, M.D., and Shahar Kol, M.D. \\ In Vitro Fertilization-Haifa, Elisha Hospital, Haifa, Israel
}

Hypogonadotropic hypogonadism $(\mathrm{HH})$ is one of the least common etiologies for female infertility, although treatment in most cases leads to successful conception. The development of urinary-derived gonadotropins (hMG) a few decades ago paved the way to replacing the absent endogenous hormones $(1,2)$. Other alternatives to therapy include native GnRH or recombinant FSH, although the use of the time-honored hMG preparations leads in most cases to ovulation (3). It is of interest that the average treatment duration and the number of ampules used are higher compared with patients with other etiologies of infertility (2). This may be explained by the "dormant" ovaries that need to be primed before follicular response is achieved.

In most cases, the diagnosis of $\mathrm{HH}$ is made at an early age, and when pregnancy is desired, hMG treatment is initiated. In most cases, the patient has a good chance of becoming pregnant as often as she wants to. Occasionally, the patient may present for treatment at a more advanced age. This may occur in cases of remarriage or because of male factor infertility in addition to the female condition.

The usual predictors of ovarian reserve (cycle day $3 \mathrm{FSH}$ or clomiphene citrate challenge test) are not applicable in this situation; therefore, there is no way to predict the patient response to ovarian stimulation. In these situations the question arises at which dose to start stimulation and when lack of potential response can be defined.

To the best of our knowledge, the question remains unanswered by the available medical literature. We present a case that vividly illustrates the concept that one should not use routine procedures for patients who have low (or no) response. Using a high hMG dose for an unusually long time may be rewarding. (Fertil Steril ${ }^{\circledR} 2000 ; 74: 401-2$. (2000 by American Society for Reproductive Medicine.)

\section{CASE REPORT}

A 40-year-old patient was referred for IVF treatment. Hypogonadotropic hypogonadism was diagnosed at 16 years of age, after investigation for primary amenorrhea. Fertility work-up revealed a normal hysterogram and a normal pelvis at laparoscopy. Karyotype analysis revealed a pericentric chromosome 2 inversion (estimated to occur in up to $1 \%$ of the population, probably without clinical relevance to $\mathrm{HH}$ ). Before her referral, repeated efforts with induction of ovulation with $\mathrm{hMG}$ were conducted for $>13$ years. Ovulation was accomplished with the use of four to five hMG ampules daily. However, pregnancy was not achieved despite the use of intrauterine insemination. The patient's partner had a sperm concentration of $11 \times 10^{6} / \mathrm{mL}$, of which $44 \%$ were motile and $57 \%$ were abnormal.

During the first treatment cycle, ovarian stimulation with four ampules of hMG (Pergonal; Teva, Petach-Tikva, Israel) was discontinued after 25 days because of lack of response $\left(\mathrm{E}_{2}\right.$ level of $\left.<73 \mathrm{pmol} / \mathrm{L}\right)$. During the second stimulation cycle, the dose was increased to six ampules a day. After 20 stimulation days (120 ampules), a single oocyte was retrieved and fertilized by intracytoplasmic sperm injection (ICSI), and a four-cell embryo was replaced; however, only "biochemical" pregnancy was achieved (the $\beta$-hCG level rose to $42 \mathrm{IU} / \mathrm{L})$. A repeated cycle with six ampules a day yielded similar results after 14 stimulation days.

It was then decided to increase the stimulation dose to eight ampules per day. At that starting dose, and after $106 \mathrm{hMG}$ ampules were given during 15 days, four metaphase II oocytes were retrieved, all of which were subjected to ICSI. Normal fertilization was noted for all injected oocytes. Embryo transfer of all four embryos resulted in a clinical pregnancy of twins. Spontaneous delivery at 37 weeks' gestational age resulted in the birth of twins $(2,370 \mathrm{~g}$ and $2,400 \mathrm{~g})$.

$0015-0282 / 00 / \$ 20.00$ PII S0015-0282(00)00599-9 


\section{DISCUSSION}

The relatively high number of hMG ampules needed to induce ovulation in an $\mathrm{HH}$ female patient was well documented (2); however, the prognosis for inducing ovulation in these patients is favorable. A specific prediction of an individual patient to respond to hMG treatment cannot be based on baseline LH and FSH levels, which we usually use for other patients. Only after a patient is subjected to such treatment can we estimate her pattern of response.

A pattern of relatively low response to $\mathrm{hMG}$ treatment was established for our patient before her IVF treatments because four to five ampules were needed to induce ovarian response. Pregnancy was not achieved, probably because of the male factor. Over time, her ovarian responsiveness deteriorated even further, because it took eight ampules of hMG daily to induce an ovarian response.

We must know how high a dose of hMG to give and how long to give this dose before we give up. Ferrari and Crosignani (4) used massive doses of hMG therapy in a patient with $\mathrm{HH}$ after head trauma before giving up. The current case report clearly illustrates that in this specific group of patients, it is worthwhile to go to extremes in treatment duration and dose.
These extremes are, of course, not relevant for patients with other etiologies for infertility. However, in female patients with $\mathrm{HH}$ the threshold for ovarian response may differ substantially from that established for other patients. To the best of our knowledge, the age-dependent decline in ovarian response for patients with $\mathrm{HH}$ has not been established.

We conclude that patients with $\mathrm{HH}$ undergoing ovarian stimulation for IVF should be carefully assessed, on a trial and error basis, for ovarian response before we give up on obtaining fertilizable oocytes.

\section{References}

1. Sungurtekin U, Fraser IS, and Shearman RP. Pregnancy in women with Kallmann's syndrome. Fertil Steril 1995;63:494-9.

2. Spitz IM, Rosen E, Ben-Aderet N, Polishuk W, Jaffe H, Bercovici B. Isolated hypogonadotropic hypogonadism: induction of ovulation with exogenous gonadotropins. Fertil Steril 1977;28:535-40.

3. Shoham Z, Balen A, Patel A, Jacobs HS. Results of ovulation induction using human menopausal gonadotropin or purified follicle-stimulating hormone in hypogonadotropic hypogonadism patients. Fertil Steril 1991; $56: 1048-53$

4. Ferrari C, Crosignani PG. Ovarian failure without gonadotropin elevation in patient with post-traumatic isolated hypogonadotropic hypogonadism. Eur J Obstet Gynecol Reprod Biol 1986;21:241-4. 